\title{
MicroRNA-126 inhibits proliferation and metastasis in prostate cancer via regulation of ADAM9
}

\author{
YIBO HUA $^{1 *}$, CHAO LIANG $^{1 *}$, CHENKUI MIAO $^{1 *}$, SHANGQIAN WANG $^{1}$, SHIFENG SU $^{1}$, \\ PENGFEI SHAO ${ }^{1}$, BIANJIANG LIU ${ }^{1}$, MEILING BAO ${ }^{2}$, JUNDONG ZHU ${ }^{1}$, AIMING XU ${ }^{1}$, \\ JIANZHONG ZHANG ${ }^{1}$, JIE LI ${ }^{1}$ and ZENGJUN WANG ${ }^{1}$ \\ Departments of ${ }^{1}$ Urology and ${ }^{2}$ Pathology, The First Affiliated Hospital of Nanjing Medical University, \\ Nanjing, Jiangsu 210029, P.R. China
}

Received December 1, 2016; Accepted February 27, 2018

DOI: $10.3892 / 01.2018 .8528$

\begin{abstract}
The aberrant expression of microRNAs (miRs) has been identified to serve a crucial role in tumor progression. The present study aimed to evaluate the role of miR-126 in human prostate cancer (PCa). Firstly, miR-126 expression in prostate cancer tissues and cell lines was analyzed. A luciferase reporter assay and a rescue assay were performed, which identified ADAM metalloproteinase domain 9 (ADAM9) as the target gene of miR-126. Subsequently, Kaplan-Meier and log-rank analyses were used to investigate the association between ADAM9 expression and PCa prognosis. The results revealed that miR-126 expression was significantly downregulated in PCa tissues and cell lines. miR-126 overexpression was demonstrated to reduce PCa cell proliferation and metastasis, and to reverse the epithelial-mesenchymal transition process in vitro. In addition, as the target gene of miR-126, the upregulation of ADAM9 reestablished cell functions, including cell proliferation, migration and invasion. Patients with high ADAM9 expression levels exhibited a shorter biochemical recurrence-free survival time. In summary, miR-126 serves a role in the proliferation and metastasis of PCa cells, indicating that miR-126 and ADAM9 may represent potential biomarkers in the progression of advanced $\mathrm{PCa}$, in addition to therapeutic targets.
\end{abstract}

Correspondence to: Dr Zengjun Wang or Dr Jie Li, Department of Urology, The First Affiliated Hospital of Nanjing Medical University, 300 Guangzhou Road, Nanjing, Jiangsu 210029, P.R. China

E-mail: zengjunwang@njmu.edu.cn

E-mail: 1ijie_urology@163.com

Abbreviations: PCa, prostate cancer; EMT, epithelial-mesenchymal transition; TMA, tissue microarray; FBS, fetal bovine serum; RT-qPCR, reverse transcription-quantitative polymerase chain reaction; UTR, untranslated region; CCK-8, Cell Counting Kit-8

*Contributed equally

Key words: miR-126, ADAM metalloproteinase domain 9, prostate cancer, proliferation, metastasis, epithelial-mesenchymal transition

\section{Introduction}

Prostate cancer ( $\mathrm{PCa}$ ) is the second highest cause of cancer-associated mortality in males, particularly in Western countries (1). In recent years, the morbidity and mortality of PCa have been increasing in Chinese males (2). The 5-year survival rate of patients with localized $\mathrm{PCa}$ is $\sim 100 \%$, due to the availability of effective treatments (3). However, certain patients experience progression to metastatic castrate-resistant prostate cancer, which is the final stage and has a poor prognosis, eventually resulting in mortality (4). Therefore, identifying accurate biomarkers for the early diagnosis of PCa, as well as efficient targets for the therapy of advanced PCa, is urgently required.

MicroRNAs (miRNAs/miRs) are a group of short and non-coding RNA sequences that serve an important role in the post-transcriptional regulation of gene expression (5). Previous studies have demonstrated that miRNAs may act as tumor suppressors or oncogenes during tumor development, and that the aberrant expression of certain miRNAs is closely associated with the progression of various types of cancer $(6,7)$. Several studies have reported that the expression of miR-126 is suppressed in multiple cancer types, and that it may act as a tumor suppressor gene by inhibiting a number of potential oncogenes and signaling pathways (8). In 2013, Feng et al (9) reported that miR-126 suppresses colon cancer progression via negatively regulating the expression of C-X-C chemokine receptor type 4 . CRK was also confirmed as a direct target of miR-126 in gastric carcinoma (9) and lung cancer (10); this gene has a key role in various signaling pathways regulating cell adhesion, proliferation and migration $(11,12)$. In non-small cell lung cancer, activity of the phosphoinositide 3-kinase-AKT pathway was suppressed by miR-126 (13). According to a previous study, miR-126 expression may serve as an independent prognostic predictor in patients with $\mathrm{PCa}$ subsequent to radical prostatectomy (14). Another study revealed that miR-126 could inhibit invasion and proliferation by targeting phosphoinositide-3-kinase regulatory subunit 2 in $\mathrm{PCa}$ cells (15). In the current study, it was identified that miR-126 can reduce the expression of the ADAM metalloproteinase domain 9 (ADAM9) protein, a member of the zinc metalloprotease class, which is involved in the development of various types 
of tumors (16). In addition, ADAM9 has been demonstrated to modulate the epithelial phenotype (17). In the current study, the role of the miR-126/ADAM9 axis in the invasion, proliferation, colony formation and epithelial-mesenchymal transition (EMT) of PCa cells was demonstrated. These revelations may improve the understanding of miR-126 in PCa progression, with miR-126 potentially becoming a novel molecular target for advanced PCa treatment.

\section{Materials and methods}

Patients and tissue microarrays (TMAs). A total of $93 \mathrm{PCa}$ tissues were collected from patients with a mean age of 69.9, ranging from 56 to 81 years old who underwent radical prostatectomy between May 2008 and December 2011 at The First Affiliated Hospital of Nanjing Medical University (Nanjing, China). All recruited patients provided informed consent prior to recruitment. The Ethics Committee of The First Affiliated Hospital of Nanjing Medical University approved the current study, including the protocols. The clinical information of the 93 patients was summarized in Table I, and the deadline for follow-up was April 2016. Triplicate tissue cores with diameters of $0.6 \mathrm{~mm}$ were extracted from each PCa tissue.

Immunohistochemistry. Xylene was used to deparaffinize serial sections from TMA blocks, which were then rehydrated through an ethanol gradient; the serial sections were then blocked for $10 \mathrm{~min}$ in hydrogen peroxide diluted $(3 \%)$ in methanol. For antigen retrieval, sections were incubated in a steam pressure cooker $\left(210^{\circ} \mathrm{C}\right)$, for $2 \mathrm{~min}$ in citrate buffer (10 ml, pH 6.0). Subsequently, the samples were blocked for $5 \mathrm{~min}$, then incubated overnight at $4^{\circ} \mathrm{C}$ with primary antibodies against ADAM9 (cat. no. ab186833; 1:100; Abcam, Cambridge, UK). The samples were washed for $10 \mathrm{~min}$ in PBS and incubated with the peroxidase-conjugated goat anti-rabbit $\operatorname{IgG}(\mathrm{H}+\mathrm{L})$ secondary antibody (dilution, 1:1,000; cat. no. ZB-2301; ZSGB-BIO, Inc., Beijing, China) at $37^{\circ} \mathrm{C}$ for $30 \mathrm{~min}$. Following another wash with PBS for $10 \mathrm{~min}$ visualization (fluorescence microscope; x100 and x400 magnification) of the antibody reaction was conducted with a fresh substrate solution containing 3,3'diaminobenzidine. The sections were counterstained with hematoxylin at room temperature for 2 min, dehydrated and coverslipped.

Evaluation of staining. Evaluation of immunohistochemical staining was conducted by two experienced pathologists blinded to the clinical data. The expression of ADAM9 was observed in the cytoplasm of tumor cells at different intensities. The staining intensity was evaluated with a semi-quantitative scoring system, as follows: 0 , negative staining; 1 , weak staining; 2 , moderate staining; and 3, strong staining. The final immunohistochemical scores of each $\mathrm{PCa}$ tissues were obtained by multiplying the percentage of tissue stained (0-100\%) and the corresponding intensity grade, resulting in a score of 0-300. To distinguish between low and high levels of ADAM9 expression, the median score was used as a cut-off value.

Cell culture and human clinical samples. Human androgen-independent prostate cancer cell lines (DU145 and PC3), normal prostate epithelial cells (RWPE-1) and 293T cells were purchased from the Chinese Academy of Sciences (Beijing, China). DU145 and PC3 cell lines were cultured in F-12K medium (Gibco; Thermo Fisher Scientific, Inc., Waltham, MA, USA). RWPE-1 and 293T cells were cultured in Dulbecco's modified Eagle's medium (Gibco; Thermo Fisher Scientific, Inc.). The medium was supplemented with $10 \%$ fetal bovine serum (FBS; Gibco; Thermo Fisher Scientific, Inc.), and cells well incubated in a humidified air atmosphere at $37^{\circ} \mathrm{C}$ and $5 \% \mathrm{CO}_{2}$.

A total of 18 pairs of PCa tissues and corresponding adjacent normal tissues were obtained from patients with a mean age of 68.1 years old, ranging from 57-77 years old, who underwent radical prostatectomy between March 2014 and November 2014, with informed consent, at The First Affiliated Hospital of Nanjing Medical University. The pathologist confirmed their histological characteristics, and the clinical characteristics were summarized in Table II.

RNA extraction and reverse transcription-quantitative polymerase chain reaction $(R T-q P C R)$. The RNA was extracted from cell lines and tumor samples using TRIzol ${ }^{\circledR}$ reagent (Invitrogen; Thermo Fisher Scientific, Inc.). miRNA was reversed transcribed into cDNA using $\mathrm{MiR}^{\mathrm{T}} \mathrm{X}^{\mathrm{TM}}$ miRNA First-Strand Synthesis (Takara Biotechnology Co., Ltd., Kusatsu, Japan). Total RNA was reversed transcribed into cDNA using PrimeScript RT Master Mix (Takara Biotechnology Co., Ltd.). RT-qPCR was performed using FastStart Universal SYBR Green Master (Roche Diagnostics, Indianapolis, IN, USA) with the following thermal cycling protocol: $95^{\circ} \mathrm{C}$ for $5 \mathrm{~min}, 95^{\circ} \mathrm{C}$ for $10 \mathrm{sec}$ and $60^{\circ} \mathrm{C}$ for $20 \mathrm{sec}$, for 40 cycles. U6 was used as the internal control for miR-126, and $\beta$-actin was used as the internal control for ADAM9. The primers for miR-126 were designed by and purchased from Tiangen Biotech Co., Ltd. (Beijing, China). The following primer sequences for miR-126 were used: Forward, 5'-CAG TGCGTGTCGTGGAGT-3' and reverse, 5'-GGGGCGTAC CGTGAGTAAT-3' (Realgene Biotech, Inc., Nanjing, China). The following primer sequences for U6 were used: Forward, 5'-CGCTTCACGAATTTGCGTGTCAT-3' and reverse, 5'-GCTTCGGCAGCACATATACTAAAAT-3' (Realgene Biotech, Inc.). The following primer sequences for ADAM9 were used: Forward 5'-CCCCCAAAT TGTGAGACT AAAG-3' and reverse, 5'-TCCCGTCCCTCAATGCAGTAT-3' (Realgene Biotech, Inc.). The following primer sequences for $\beta$-actin were used: Forward 5'-TGACGGGGTCACCCACAC TGTGCCCATCTA-3' and reverse, 5'-CTAGAAGCATTT GCGGTGGACGATGGAGGG-3' (Realgene Biotech, Inc.). The $2^{-\Delta \Delta \mathrm{Cq}}$ method was used to analyze the relative gene expression (18).

Transient transfection. The hsa-miR-126-3p mimics, non-specific miRNA control (negative control), ADAM9 siRNA and scrambled siRNA (negative control) were designed and chemically synthesized by Shanghai GenePharma Co., Ltd. (Shanghai, China). The following sequences of siRNA for ADAM9 were used: Forward, 5'-CCAGAGAAGUUC CUAUAUA-3', and reverse, 5'-UAUAUAGGA ACUUCU CUGG-3'. The following sequences of scrambled siRNA were used: Forward, 5'-UUCUCCGAACGUGUCACGUTT-3', 
Table I. Association of ADAM9 expression and clinicopathological characteristics of the patients.

\begin{tabular}{|c|c|c|c|c|}
\hline \multirow[b]{2}{*}{ Variable } & \multirow[b]{2}{*}{ Total, n } & \multicolumn{3}{|c|}{ ADAM9 expression, $\mathrm{n}(\%)$} \\
\hline & & Low $(n=42)$ & $\operatorname{High}(n=51)$ & $\mathrm{P}$-value \\
\hline Age, years & & & & 0.381 \\
\hline$<60$ & 6 & $3(50.0)$ & $3(50.0)$ & \\
\hline $60-70$ & 42 & $22(52.4)$ & $20(47.6)$ & \\
\hline$>70$ & 45 & $17(37.8)$ & $28(62.2)$ & \\
\hline Preoperative PSA (ng/ml) & & & & 0.083 \\
\hline$<10$ & 27 & $17(63.0)$ & $10(37.0)$ & \\
\hline $10-20$ & 30 & $12(40.0)$ & $18(60.0)$ & \\
\hline$>20$ & 36 & $13(36.1)$ & $23(63.9)$ & \\
\hline Gleason score & & & & 0.853 \\
\hline$<7$ & 55 & $26(47.3)$ & $29(52.7)$ & \\
\hline$=7$ & 20 & $8(40.0)$ & $12(60.0)$ & \\
\hline$>7$ & 18 & $8(44.4)$ & $10(55.6)$ & \\
\hline T stage & & & & 0.564 \\
\hline pT2 & 79 & $37(46.8)$ & $42(53.2)$ & \\
\hline $\mathrm{pT} 3 / \mathrm{T} 4$ & 14 & $5(35.7)$ & $9(64.3)$ & \\
\hline Biochemical recurrence & & & & $0.030^{\mathrm{a}}$ \\
\hline Negative & 59 & $32(54.2)$ & $27(45.8)$ & \\
\hline Positive & 34 & $10(29.4)$ & $24(70.6)$ & \\
\hline
\end{tabular}

${ }^{\mathrm{a}} \mathrm{P}<0.05$. P-values were two-tailed and based on the Pearson's $\chi^{2}$ test.

Table II. Characteristics of the 18 patients treated by radical prostatectomy.

\begin{tabular}{lcr}
\hline Variable & Total, $\mathrm{n}$ & $\mathrm{n}, \%$ \\
\hline Age & & \\
Mean \pm SD (year) & $68.1 \pm 6.6$ & \\
$<60$ & 1 & 5.6 \\
$60-70$ & 8 & 44.4 \\
$>70$ & 9 & 50.0 \\
Gleason score & & \\
$<7$ & 13 & 72.2 \\
$=7$ & 2 & 11.1 \\
$>7$ & 3 & 16.7 \\
T stage & & \\
pT2 & 14 & 77.8 \\
pT3/T4 & 4 & 22.2 \\
\hline
\end{tabular}

$\mathrm{SD}$, standard deviation.

and reverse, 5'-ACGUGACACGUUCGGAGAATT-3'. PCa cells were maintained until mid-log phase, then digested with trypsin and seeded into 6-well plates at a density of $1 \times 10^{5}$ cells/well. Following culture for $48 \mathrm{~h}$, when cells reached $60 \%$ confluence, cells were processed for transfection. PCa cells were transfected with $2 \mu \mathrm{g}$ miR-126 mimics or negative control using Lipofectamine ${ }^{\circledR} 2000$ (Invitrogen; Thermo Fisher Scientific, Inc.) in Opti-MEM medium, according to the manufacturer's protocol. In the same way, $2.5 \mu \mathrm{g}$ ADAM9 siRNA or scrambled siRNA were transfected into PCa cells with Lipofectamine ${ }^{\circledR} 2000$. Following transfection for 4-6 h, the medium surrounding the cells was replaced with F12-K.

ADAM9 overexpression plasmids were constructed by Obio Technology Co., Ltd. (Shanghai, China). For co-transfection, Lipofectamine ${ }^{\circledR} 3000$ (Invitrogen; Thermo Fisher Scientific, Inc.) was used, and the PCa cells were divided into two groups: One group was co-transfected with $2 \mu \mathrm{g}$ miR-126 mimics and $5 \mu \mathrm{g}$ pLenti-ADAM9, while the other group was co-transfected with $2 \mu \mathrm{g}$ non-specific miRNA control and $5 \mu \mathrm{g}$ empty plasmids (Obio Technology Co., Ltd.).

Luciferase reporter assay. The binding site of miR-126 in ADAM9 was predicted by TargetScan (www.targetscan. org) and miRanda (www.microrna.org). To construct a luciferase reporter plasmid with wild type ADAM9, the 3' untranslated region (UTR) of ADAM9, predicted to bind with miR-126, was chemosynthesized, amplified and cloned into a pMIR-REPORT luciferase vector (Obio Technology Co., Ltd.). The binding sequence was point-mutated to form a control luciferase reporter plasmid by Obio Technology Co., Ltd.. For the luciferase reporter assay, the 293T cells were co-transfected with miR-126 mimics or the miRNA control, and the wild type or mutant-type ADAM9 plasmid. After incubation for $48 \mathrm{~h}$, a Dual-Luciferase ${ }^{\circledR}$ Reporter 
assay system (Promega Corporation, Madison, WI, USA) was used to determine luciferase activity on the LD400 Luminometer (Beckman Coulter, Inc., Brea, CA, USA). Renilla luciferase activity was normalized to firefly luciferase activity.

Cell proliferation assays. Cell proliferation was assessed using a Cell Counting Kit-8 (CCK-8) and colony formation assays. For the CCK-8 assay, $48 \mathrm{~h}$ subsequent to transfection, PC3 and DU145 cells were seeded in 96-well plates at a density of 2,000 cells/well. CCK-8 (Dojindo Molecular Technologies, Inc., Kumamoto, Japan) was used to determine cell proliferation at 24, 48, 72 and $96 \mathrm{~h}$ after seeding. Absorbance was determined at $450 \mathrm{~nm}$ by an Infinite M200 Pro absorbance reader (Thermo Fisher Scientific, Inc.).

For the colony formation assay, at $48 \mathrm{~h}$ after transfection, PC3 and DU145 cells were harvested and plated in cell culture dishes at a density of 1,000 cells/dish. Following incubation at $37^{\circ} \mathrm{C}$ for 14 days, the cells were stained with $0.005 \%$ crystal violet for $2 \mathrm{~h}$; the colonies were then counted under a fluorescence-inverted microscope (x100 magnification).

Transwell migration and invasion assays. The in vitro migration and invasion assays were performed using 24-well chambers with $8-\mu \mathrm{m}$ pore size PET track-etched membranes (Corning, Inc., Corning, NY, USA). After transfection for $48 \mathrm{~h}$, a total of $5 \times 10^{4}$ transfected cells were suspended in the upper chamber with serum-free medium, while the lower chamber was filled with $20 \%$ FBS-containing medium. The membranes were covered with Matrige ${ }^{\circledR}$ (BD Biosciences, San Jose, CA, USA) to form matrix barriers in the invasion assays. After incubation at $37^{\circ} \mathrm{C}$ for 24 (migration assay) or $48 \mathrm{~h}$ (invasion assay), a cotton swab was used to remove the cells on the upper surface, while the cells on the bottom of the membranes were fixed in $4 \%$ methanol solution for $1 \mathrm{~h}$ and stained with $0.005 \%$ crystal violet for $2 \mathrm{~h}$. These were then counted in three random fields under a fluorescence-inverted microscope (x200 magnification).

Western blot assay. Total proteins were extracted from tumor cells using protein lysis buffer (Beyotime Institute of Biotechnology, Nantong, Jiangsu, China). The protein concentration was measured using a BCA kit (Beyotime Institute of Biotechnology), according to the manufacturer's protocols. For western blot analysis, $25 \mu \mathrm{g}$ of each protein were separated via $10 \%$ SDS-PAGE. Subsequently, the proteins were transferred to PVDF membranes in transfer buffer, which contains Tris-HCL SDS, Glycine and methanol. The membranes were then incubated with monoclonal rabbit anti-ADAM9 (dilution, 1:1,000; cat. no. ab186833; Abcam), monoclonal rabbit anti-E-cadherin (dilution, 1:1,000; cat. no. 3195), monoclonal rabbit anti-N-cadherin (dilution, 1:1,000; cat. no. 13116) or monoclonal rabbit anti-Vimentin (dilution, 1:1,000; cat. no. 5741) (Cell Signaling Technology, Inc., Danvers, MA, USA), all at $4^{\circ} \mathrm{C}$ overnight; rabbit monoclonal $\beta$-actin (Abcam) served as the internal control. Following this, the membranes were washed three times in TBST and incubated with an anti-rabbit secondary antibody solution (dilution, 1:4,000) at room temperature for $2 \mathrm{~h}$. The indicated proteins were detected using an enhanced chemiluminescence western blotting detection system (Bio-Rad ChemiDoc XRS+). The relative protein expression was analyzed by Image-Lab software (version 6.0; Bio-Rad Laboratories, Inc., Hercules, CA, USA), represented as the density ratio vs. actin.

Statistical analyses. The associations between protein expression values and clinicopathological factors were assessed using the $\chi^{2}$-test. Kaplan-Meier curves and log-rank tests were used to assess the association of ADAM9 expression with recurrence-free survival. Results are expressed as the mean \pm standard deviation. Differences between two or three groups were compared with the Student's t-test or one-way analysis of variance followed by Dunnett's t-test. A value of $\mathrm{P} \leq 0.05$ was considered to indicate a statistically significant difference. All statistical calculations were performed using SPSS v.13.0 software (SPSS, Inc., Chicago, IL, USA).

\section{Results}

miR-126 expression is downregulated in PCa tissues and cell lines. The expression levels of miR-126 in 36 frozen samples from patients with $\mathrm{PCa}$ (18 cancerous and 18 corresponding adjacent tissues) were examined by RT-qPCR. The results indicated that, compared with adjacent normal tissues, miR-126 expression is significantly reduced $(\mathrm{P}<0.01)$ in $\mathrm{PCa}$ tissues (Fig. 1A). Similarly, RT-qPCR was performed to determine miR-126 expression levels in two PCa cell lines (PC3, DU145) and normal prostate epithelial cells (RWPE-1). As depicted in Fig. 1B, the expression level of miR-126 was significantly lower in PC3 and DU145, compared with in RWPE-1 cells $(\mathrm{P}<0.01)$. These results indicated that miR-126 may function as a suppressive gene in the progression of $\mathrm{PCa}$.

miR-126 overexpression suppresses PCa cell proliferation, metastasis and the EMT process. To investigate the effect of miR-126 in PCa cell function, miR-126 mimics were used to overexpress miR-126 in PC3 and DU145 cells (Fig. 1C). Subsequently, a CCK-8 assay was performed to evaluate the cell proliferation. It was observed that miR-126 overexpression significantly suppressed the proliferation rate of PC3 and DU145 cells at 72 or $96 \mathrm{~h}$ (Fig. 1D). Consistently, the same trend in colony formation assays (Fig. 1E) was also noticed. These results suggested that miR-126 overexpression decreased $\mathrm{PCa}$ cell growth.

Transwell assays were performed to assess the significance of miR-126 in PCa cell metastasis. As demonstrated in Fig. $1 \mathrm{~F}$ and $\mathrm{G}$, the migration and invasion rates were significantly inhibited after transfecting miR-126 mimics into PC3 and DU145 cells $(\mathrm{P}<0.01)$. This suggested that miR-126 may function as a suppressor in PCa metastasis.

EMT is a crucial process during tumor metastasis. Based on the association between miR-126 expression and the metastasis of PCa cells, a western blot assay was performed to assess EMT-associated protein expression. Following transfection with miR-126 mimics, increased expression of the epithelial marker E-cadherin, and decreased expression of the mesenchymal markers $\mathrm{N}$-cadherin and Vimentin (Fig. 1H) were observed; however, N-cadherin was only expressed in PC3, and not in DU145 cells, which is consistent with the results 

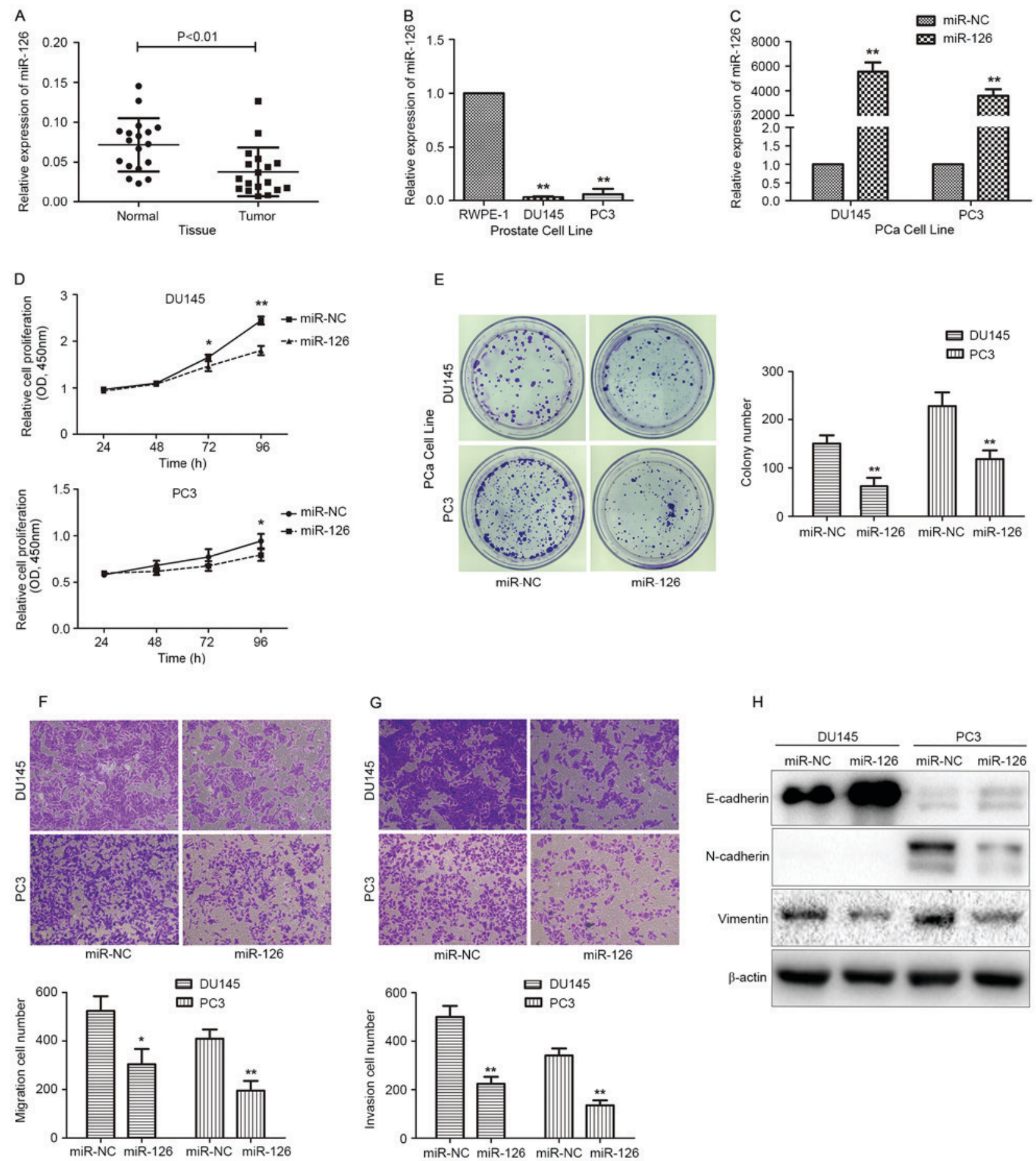

Figure 1. Expression of miR-126 was significantly lower in PCa tissues and cell lines, and miR-126 overexpression inhibited PCa cell functions. (A) Expression of miR-126 in PCa and adjacent normal tissues. (B) Expression of miR-126 in normal prostate epithelial cells (RWPE-1) and PCa cells (DU145 and PC3). (C) Reverse transcription-quantitative polymerase chain reaction was performed to validate the efficacy of overexpressing miR-126 in DU145 and PC3. The proliferation of DU145 and PC3 cells transfected with control miRNA or miR-126 mimics were analyzed by (D) Cell Counting Kit-8 assays and (E) colony formation assays. Representative results of (F) migration and $(\mathrm{G})$ invasion of aforementioned cells were analyzed by Transwell assays (magnification, $\mathrm{x} 100$ ). (H) Western blotting was performed to assess epithelial-mesenchymal transition-associated protein levels including E-cadherin, N-cadherin and Vimentin. ${ }^{*} \mathrm{P}<0.05$ and ${ }^{* *} \mathrm{P}<0.01$, Student's t-test. PCa, prostate cancer; miR/miRNA, microRNA; NC, negative control; OD, optical density.

previously reported by Nalla et al (19). The results of western blot analysis for EMT indicated that miR-126 overexpression reversed the EMT process in PCa cells.

ADAM9 is a direct target gene of miR-126. In previous literature, it was revealed that miRNA regulates gene expression by binding to the 3 'UTR of target mRNAs (20). The
3'UTR of ADAM9 was predicted to be a potential target of miR-126 via comprehensive analysis using TargetScan and miRanda (Fig. 2A). To identify the influence of miR-126 on ADAM9 expression, RT-qPCR and western blot analyses were performed on PC3 and DU145 cells transfected with miR-126. As depicted in Fig. 2B and C, the mRNA and protein expression levels of ADAM9 in PC3 and DU145 cells were 

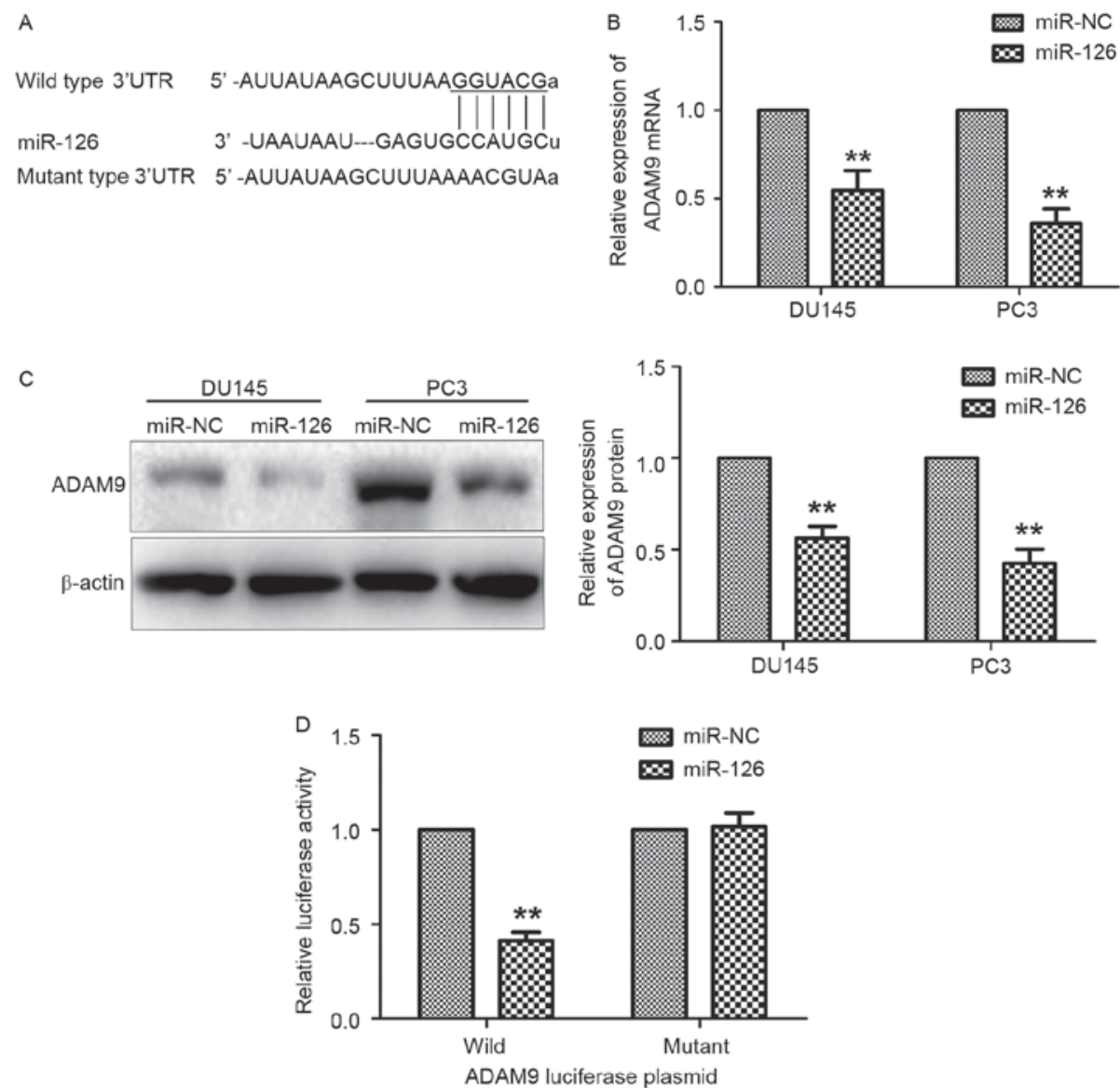

Figure 2. miR-126 directly targeted ADAM9 in PCa cells. (A) Bioinformatics analysis using TargetScan and miRanda predicted that the 3' UTR of ADAM9 contains a binding site for miR-126. ADAM9 mRNA (B) and protein (C) expression level in DU145 and PC3 cells following transfected with miR-126. (D) Relative luciferase activity in 293T cells following co-transfected with miR-126 and wild-type ADAM9 luciferase plasmid or mutant-type ADAM9 luciferase plasmid. ${ }^{* *} \mathrm{P}<0.01$, Student's t-test. UTR, untranslated region; PCa, prostate cancer; NC, negative control; miR/miRNA, microRNA; ADAM9, ADAM metalloproteinase domain 9.

significantly reduced following transfection with miR-126 mimics $(\mathrm{P}<0.01)$. To further verify the direct interaction of miR-126 and ADAM9, a luciferase reporter assay in 293T cells was conducted. As demonstrated in Fig. 2D, miR-126 overexpression decreased the luciferase activity of the wild-type ADAM9 plasmid, whereas no effects were observed with the ADAM9 mutant plasmid.

miR-126 functions as a tumor-suppressor gene by reducing ADAM9 expression. To determine whether miR-126 suppresses PCa progression through the downregulation of ADAM9 expression, the role of ADAM9 in PCa cell function was investigated. ADAM9 siRNA was transfected into DU145 and PC3 cells to decrease endogenous ADAM9 expression (Fig. 3A and B). Observations of cell proliferation (Fig. 3C), colony formation (Fig. 3D), migration (Fig. 3E) and invasion (Fig. 3F) capabilities revealed that each function was suppressed, and that the process of EMT was reversed (Fig. 3G) following the transient knockdown of ADAM9 in PCa cells. These results are consistent with those of previous experiments, which indicated the overexpression of miR-126. To further identify whether miR-126 suppresses PCa cell functions by reducing ADAM9 expression, co-transfection of ADAM9 overexpression plasmids and miR-126 mimics into PCa cells was conducted, and whether cell functions could be reversed by overexpression of ADAM9 was assessed. As depicted in Fig. 4A, ADAM9 protein was markedly increased following transfection with ADAM9 overexpression plasmids. Furthermore, restoration of ADAM9 in miR-126-overexpressing PCa cells showed no significant differences in proliferation (Fig. 4B), colony formation (Fig. 4C), migration (Fig. 4D), invasion (Fig. 4E), and the EMT process (Fig. 4F), compared with the control group.

ADAM9 may act as a prognostic indicator for PCa. The expression of ADAM9 protein in $93 \mathrm{PCa}$ tissue samples was evaluated to analyze the role of ADAM9 in PCa progression (Fig. 4G). As demonstrated by the $\chi^{2}$ test results (Table I), higher ADAM9 protein expression was significantly associated with biochemical recurrence $(\mathrm{P}=0.030)$. In addition, the Kaplan-Meier curves indicated a significantly shorter biochemical recurrence-free survival time for those patients with high ADAM9 expression ( $\mathrm{P}=0.0116$; Fig. 4H). These results indicated that high expression of ADAM9 was associated with poor prognosis in patients with $\mathrm{PCa}$.

\section{Discussion}

PCa has increasing morbidity and mortality rates, and the prognosis becomes worse as the disease develops to an 
A
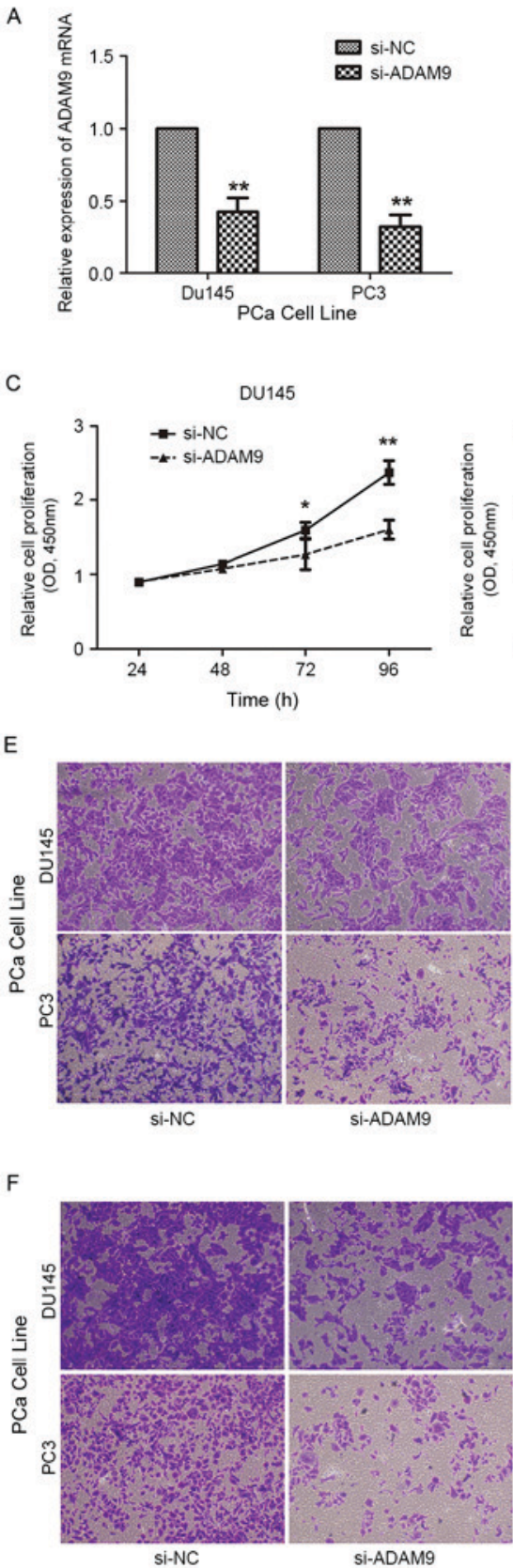

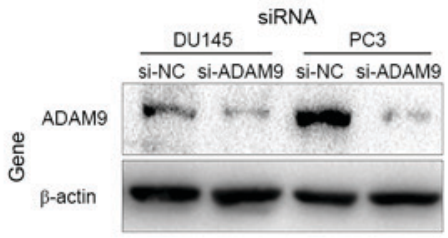

PC3
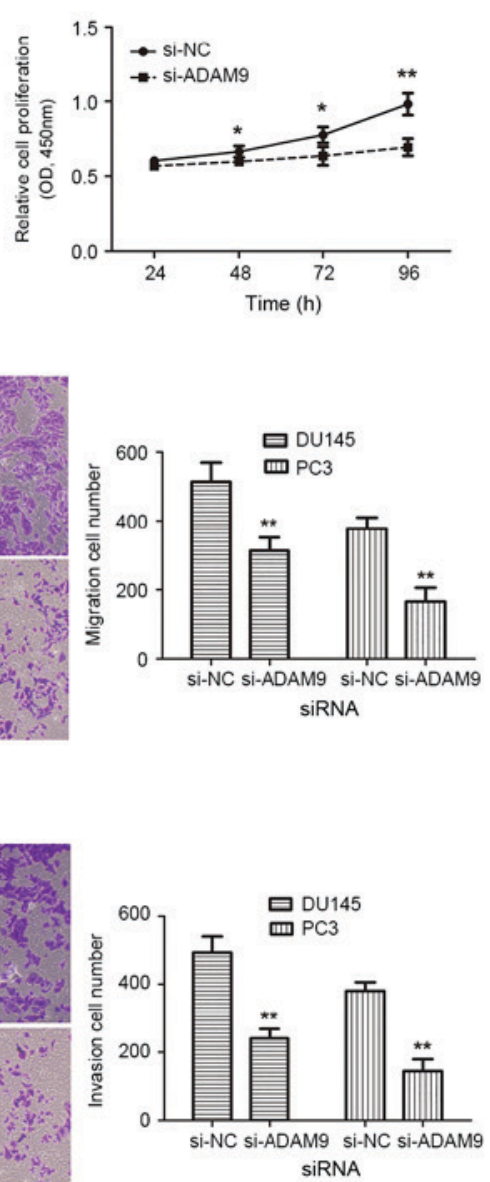

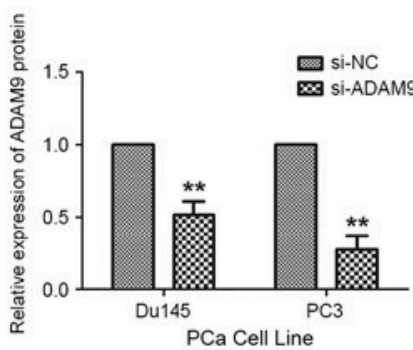

D

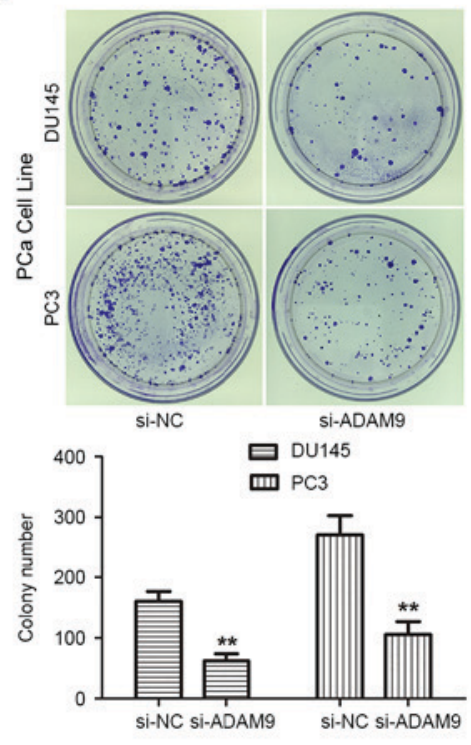

Figure 3. Knockdown of ADAM9 inhibited PCa functions. (A) Reverse transcription-quantitative polymerase chain reaction and (B) western blotting were performed to validate the efficacy of knocking down ADAM9 in DU145 and PC3 cells. The proliferation of DU145 and PC3 cells transfected with NC or ADAM9-specific siRNA were analyzed by (C) Cell Counting Kit-8 assays and (D) colony formation assays. Representative results of (E) migration and (F) invasion of aforementioned cells were analyzed by transwell assays (magnification, x100). (G) Western blotting was performed to test epithelial-mesenchymal transition associated protein including E-cadherin, $\mathrm{N}$-cadherin and Vimentin. ${ }^{*} \mathrm{P}<0.05$ and ${ }^{* *} \mathrm{P}<0.01$, Student's t-test. PCa, prostate cancer cells; EMT, epithelial-mesenchymal transition; ADAM9, ADAM metalloproteinase domain 9; NC, negative control; OD, optical density.

advanced stage or metastasizes. Various studies have demonstrated that miRNAs may function as tumor suppressors or oncogenes during tumor development, according to the roles of their target genes $(6,21)$. In PCa, several miRNAs have been reported to serve critical roles in the regulation of basic biological processes, including cell growth, apoptosis, metastasis and drug resistance $(21,22)$. These findings suggest that miRNAs have promising diagnostic and therapeutic values for patients with $\mathrm{PCa}$.

miR-126 expression is suppressed in the majority of human cancer types, and always functions as a tumor suppressor gene, to the best of our knowledge (8); however, in $\mathrm{PCa}$, the functional role and the potential prognostic value of miR-126 remain to be elucidated. In the present study, RT-qPCR was used to identify that the expression of miR-126 was significantly suppressed in PCa tissues. Studying the androgen-independent PCa cell lines PC3 and DU145 revealed that miR-126 overexpression could inhibit their proliferative and metastatic capabilities. In addition, it was verified that miR-126 functioned as the tumor suppressor by downregulating the expression of ADAM9, a member of the ADAM family. 
A
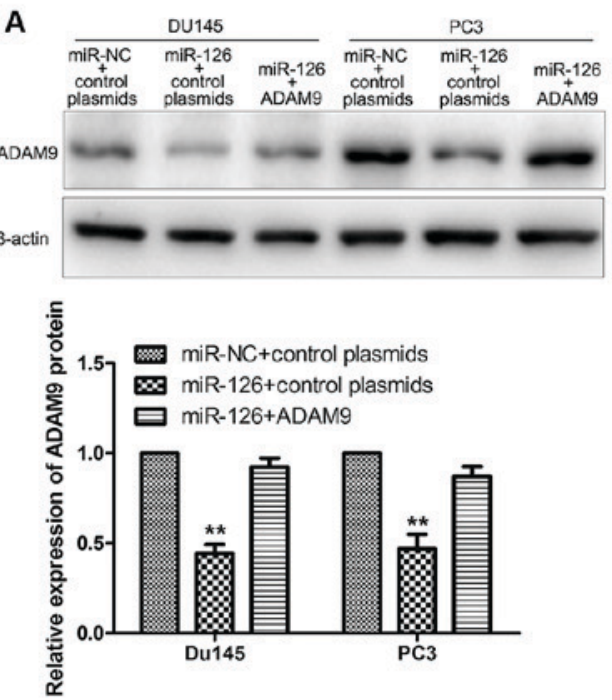

C
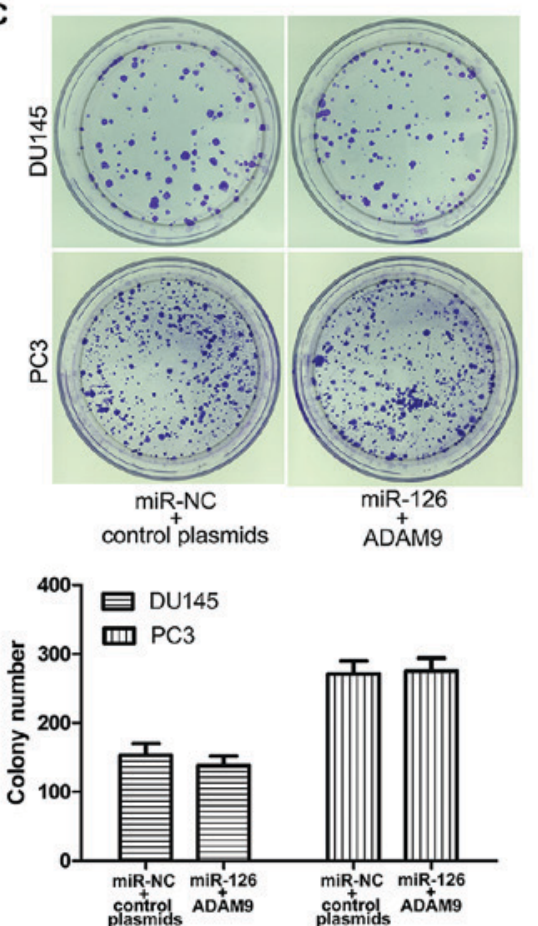

$\mathbf{F}$

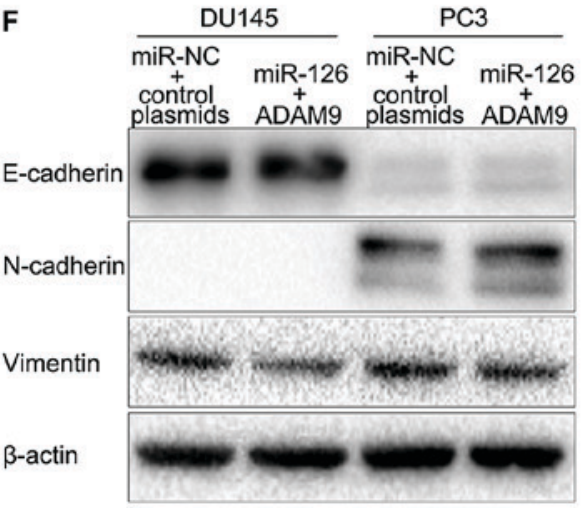

B
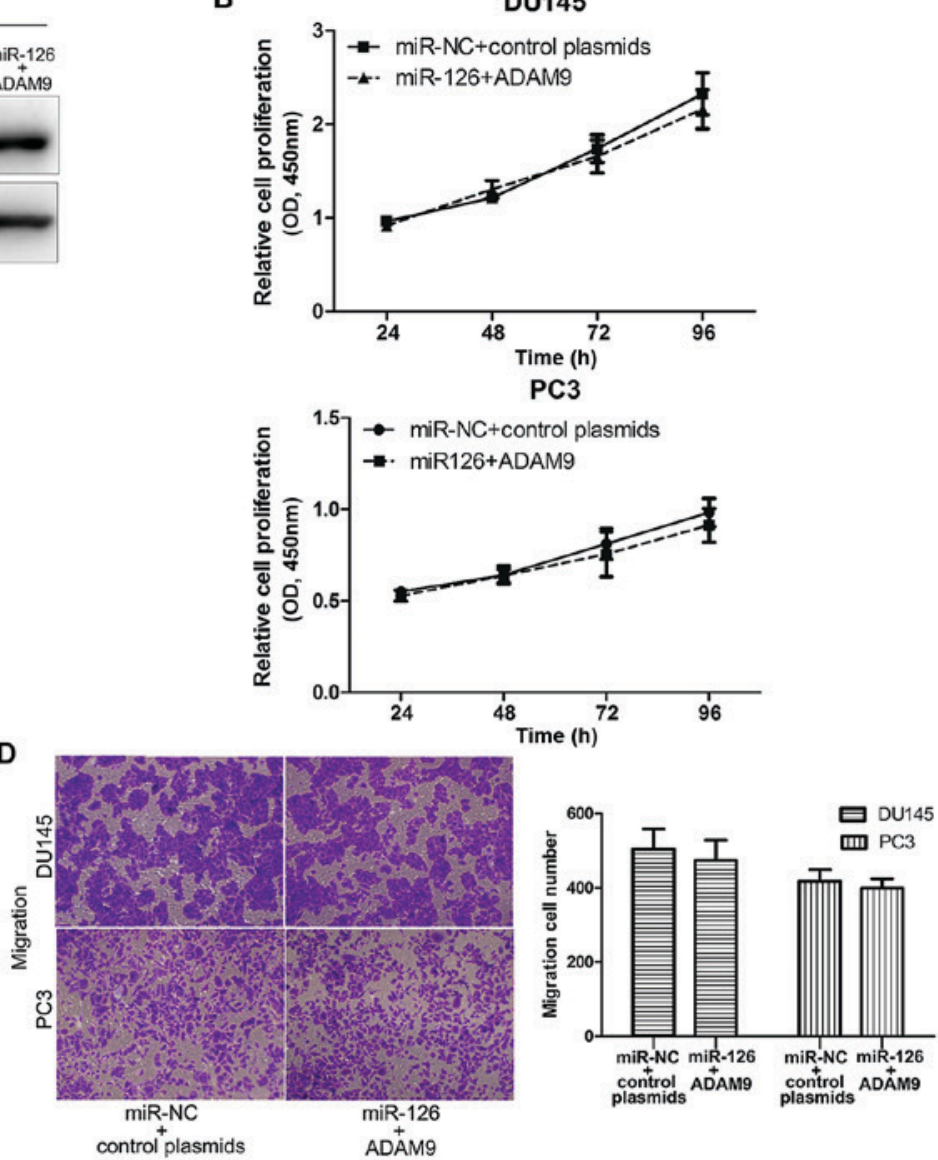

E
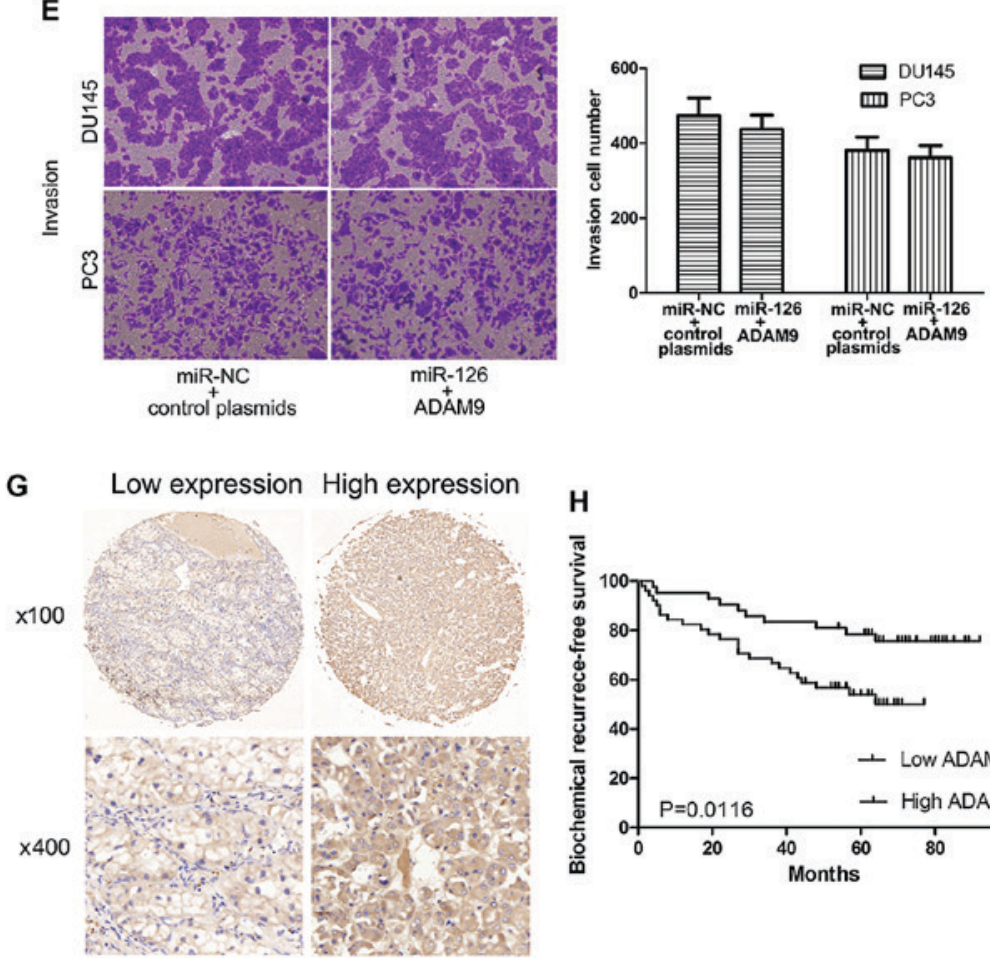

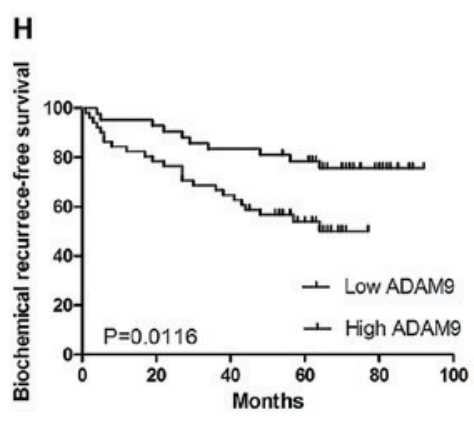

Figure 4. Re-expression of ADAM9 reestablished prostate cancer cells (PCa) cell functions and ADAM9 expression is associated with PCa prognosis. (A) Re-expression of ADAM9 was confirmed by western blot analysis following co-transfection of miR-126 mimics and pL-ADAM9. Proliferation effect of DU145 and PC 3 cells co-transfected with miR-126 mimics and pL-ADAM9 were analyzed by (B) Cell Counting Kit- 8 assays and (C) colony formation assays . Representative results of (D) migration and (E) invasion of aforementioned cells were analyzed by Transwell assays (magnification, x100). (F) Western blotting was performed to test epithelial-mesenchymal transition associated protein including E-cadherin, N-cadherin and Vimentin. (G) Immunohistochemical images of ADAM9 expression in PCa tissue microarrays. (H) Kaplan-Meier/log-rank analysis for biochemical recurrence-free survival from 93 patients with $\mathrm{PCa}$ according to ADAM9 expression in tumor tissues. ${ }^{* *} \mathrm{P}<0.01$, Student's t-test or Dunnett's t-test. PCa, prostate cancer cells; EMT, epithelial-mesenchymal transition; ADAM9, ADAM metalloproteinase domain 9; NC, negative control; OD, optical density; pL-ADAM9, pLenti-ADAM9. 
ADAMs are involved in cell adhesion, cell migration and tissue remodeling (23); these processes are important for tumor progression. Previous studies have demonstrated that various ADAMs represent promising diagnostic and prognostic markers in tumors (24). ADAM9 has been reported to be associated with cancer metastasis $(25,26)$; in addition, evidence indicates that ADAM9 serves a role in cancer proliferation by activating the epidermal growth factor receptor signaling pathway (27-29). The data from the present study indicate that miR-126 acts as a tumor suppressor by targeting ADAM9 in PCa. Previous studies have revealed that ADAM9 promotes the metastasis of cancer by regulating E-cadherin and integrins $(30,31)$. In the current study, it was revealed that miR-126 reversed the epithelial phenotype and repressed the mesenchymal phenotype via decreasing the expression of ADAM9 in PCa. EMT is a complex multistep process and serves a critical role in cancer metastasis and tumor invasion (32). Recent studies have identified that EMT serves a vital role in chemoresistance, tumor metastasis, disease aggressiveness and poor prognosis of prostate cancer $(33,34)$. Therefore, the present study suggested that miR-126 may be a novel therapeutic target to combat aggressive PCa.

To the best of our knowledge, the present study is the first to identify ADAM9 as a potential target of miR-126 in PCa cells. Furthermore, it was speculated that the reduced level of miR-126 could lead to the accumulation of ADAM9 in PCa tissues, which may result in a poor prognosis for patients with PCa. To test this hypothesis, the expression levels of ADAM9 were evaluated in TMAs constructed from 93 PCa samples. The present data suggest that ADAM9 may be a prognostic marker for the biochemical recurrence of $\mathrm{PCa}$.

In summary, the results of the current study provided evidence that miR-126 may function as a tumor-suppressive gene by targeting ADAM9 to inhibit PCa cell proliferation, migration, invasion and colony formation, as well as the EMT process. It was also identified that a high level of ADAM9 expression is an important marker for the poor prognosis of patients with $\mathrm{PCa}$. These results suggested that miR-126 may be a potential therapeutic target for the treatment of advanced $\mathrm{PCa}$.

\section{Acknowledgements}

Not applicable.

\section{Funding}

The present study was supported by the National Natural Science Foundation of China (grant nos. 81550010 and 81672532) and the Graduate International Exchange and Cooperation Projects of Nanjing Medical University (grant no. 201601C006).

\section{Availability of data and materials}

The datasets used or analyzed during the present study are available from the corresponding author on reasonable request.

\section{Authors' contributions}

Conception and design was conducted by ZW and JL. Acquisition of data was completed by YH, CL, CM, ZW and BL. Analysis and interpretation of data was conducted by SW, SS, PS, MB, AX and JZ. Drafting the manuscript was completed by YH, JZ and AX. All authors approved the final version.

\section{Ethics approval and consent to participate}

All patients gave informed consent to the study (pathological characteristics and immunohistochemical staining of $\mathrm{PCa}$ tissues obtained after curative resection and study of statistical associations with clinicopathological data). This study was approved by the medical ethics committee of the hospital and conducted in accordance with the Chinese laws and regulations.

\section{Consent for publication}

Written informed consent for the publication of any associated data and accompanying images has been obtained from all participants.

\section{Competing interests}

The authors declare that they have no competing interests.

\section{References}

1. Yamamoto S, Kawakami S, Yonese J, Fujii Y, Urakami S, Masuda H, Numao N, Ishikawa Y, Kohno A and Fukui I: Long-term oncological outcome and risk stratification in men with high-risk prostate cancer treated with radical prostatectomy. Jpn J Clin Oncol 42: 541-547, 2012.

2. Peyromaure EM, Mao K, Sun Y, Xia S, Jiang N, Zhang S, Wang G, Liu Z and Debré B: A comparative study of prostate cancer detection and management in China and in France. Can J Urol 16: 4472-4477, 2009.

3. Walz J, Gallina A, Saad F, Montorsi F, Perrotte P, Shariat SF, Jeldres C, Graefen M, Bénard F, McCormack M, et al: A nomogram predicting 10-year life expectancy in candidates for radical prostatectomy or radiotherapy for prostate cancer. J Clin Oncol 25: 3576-3581, 2007.

4. Hoang DT, Iczkowski KA, Kilari D, See W and Nevalainen MT: Androgen receptor-dependent and -independent mechanisms driving prostate cancer progression: Opportunities for therapeutic targeting from multiple angles. Oncotarget 8: 3724-3745, 2017.

5. Nana-Sinkam SP and Croce CM: Clinical applications for microRNAs in cancer. Clin Pharmacol Ther 93: 98-104, 2013.

6. Farazi TA, Hoell JI, Morozov P and Tuschl T: MicroRNAs in human cancer. Adv Exp Med Biol 774: 1-20, 2013.

7. Baer C, Claus R and Plass C: Genome-wide epigenetic regulation of miRNAs in cancer. Cancer Res 73: 473-477, 2013.

8. Ebrahimi F, Gopalan V, Smith RA and Lam AK: miR-126 in human cancers: Clinical roles and current perspectives. Exp Mol Pathol 96: 98-107, 2014.

9. Feng R, Chen X, Yu Y, Su L, Yu B, Li J, Cai Q, Yan M, Liu B and Zhu Z: miR-126 functions as a tumour suppressor in human gastric cancer. Cancer Lett 298: 50-63, 2010.

10. Crawford M, Brawner E, Batte K, Yu L, Hunter MG, Otterson GA, Nuovo G, Marsh CB and Nana-Sinkam SP: MicroRNA-126 inhibits invasion in non-small cell lung carcinoma cell lines. Biochem Biophys Res Commun 373: 607-612, 2008.

11. Kumar S, Davra V, E Obr A, Geng K, L Wood T and Birge R: Crk adaptor protein promotes PD-L1 expression, EMT and immune evasion in a murine model of triple-negative breast cancer. Oncoimmunology 7: e1376155, 2017.

12. Tsuda $\mathrm{M}$ and Tanaka S: Roles for crk in cancer metastasis and invasion. Genes Cancer 3: 334-340, 2012.

13. Yang J, Lan H, Huang X, Liu B and Tong Y: MicroRNA-126 inhibits tumor cell growth and its expression level correlates with poor survival in non-small cell lung cancer patients. PLoS One 7: e42978, 2012. 
14. Sun X, Liu Z, Yang Z, Xiao L, Wang F, He Y, Su P, Wang J and Jing B: Association of microRNA-126 expression with clinicopathological features and the risk of biochemical recurrence in prostate cancer patients undergoing radical prostatectomy. Diagn Pathol 8: 208, 2013

15. Song L, Xie X, Yu S, Peng F and Peng L: MicroRNA-126 inhibits proliferation and metastasis by targeting pik3r2 in prostate cancer. Mol Med Rep 13: 1204-1210, 2016.

16. Duffy MJ, McKiernan E, O'Donovan N and McGowan PM: Role of ADAMs in cancer formation and progression. Clin Cancer Res 15: 1140-1144, 2009.

17. Josson S, Anderson CS, Sung SY, Johnstone PA, Kubo H Hsieh CL, Arnold R, Gururajan M, Yates C and Chung LW: Inhibition of ADAM9 expression induces epithelial phenotypic alterations and sensitizes human prostate cancer cells to radiation and chemotherapy. Prostate 71: 232-240, 2011.

18. Livak KJ and Schmittgen TD: Analysis of relative gene expression data using real-time quantitative PCR and the 2(-Delta Delta C(T)) method. Methods 25: 402-408, 2001

19. Nalla AK, Estes N, Patel J and Rao JS: N-cadherin mediates angiogenesis by regulating monocyte chemoattractant protein-1 expression via PI3K/Akt signaling in prostate cancer cells. Exp Cell Res 317: 2512-2521, 2011.

20. Agostini M, Pucciarelli S, Calore F, Bedin C, Enzo M and Nitti D: miRNAs in colon and rectal cancer: A consensus for their true clinical value. Clin Chim Acta 411: 1181-1186, 2010.

21. Tian L, Fang YX, Xue JL and Chen JZ: Four microRNAs promote prostate cell proliferation with regulation of PTEN and its downstream signals in vitro. PLoS One 8: e75885, 2013.

22. Kopczyńska E: Role of microRNAs in the resistance of prostate cancer to docetaxel and paclitaxel. Contemp Oncol (Pozn) 19: 423-427, 2015.

23. Nath D, Slocombe PM, Webster A, Stephens PE, Docherty AJ and Murphy G: Meltrin gamma(ADAM-9) mediates cellular adhesion through alpha(6)beta(1 )integrin, leading to a marked induction of fibroblast cell motility. J Cell Sci 113: 2319-2328, 2000.

24. Lendeckel U, Kohl J, Arndt M, Carl-McGrath S, Donat H and Röcken C: Increased expression of ADAM family members in human breast cancer and breast cancer cell lines. J Cancer Res Clin Oncol 131: 41-48, 2005.

25. Shintani Y,Higashiyama S, Ohta M, Hirabayashi H, Yamamoto S, Yoshimasu T, Matsuda $\mathrm{H}$ and Matsuura N: Overexpression of ADAM9 in non-small cell lung cancer correlates with brain metastasis. Cancer Res 64: 4190-4196, 2004.
26. Mazzocca A, Coppari R, De Franco R, Cho JY, Libermann TA, Pinzani M and Toker A: A secreted form of ADAM9 promotes carcinoma invasion through tumor-stromal interactions. Cancer Res 65: 4728-4738, 2005

27. Kim JM, Jeung HC, Rha SY, Yu EJ, Kim TS, Shin YK, Zhang X, Park KH, Park SW, Chung HC and Powis G: The effect of disintegrin-metalloproteinase ADAM9 in gastric cancer progression. Mol Cancer Ther 13: 3074-3085, 2014.

28. Fry JL and Toker A: Secreted and membrane-bound isoforms of protease ADAM9 have opposing effects on breast cancer cell migration. Cancer Res 70: 8187-8198, 2010.

29. Itabashi H, Maesawa C, Oikawa H, Kotani K, Sakurai E, Kato K, Komatsu H, Nitta H, Kawamura H, Wakabayashi G and Masuda T: Angiotensin II and epidermal growth factor receptor cross-talk mediated by a disintegrin and metalloprotease accelerates tumor cell proliferation of hepatocellular carcinoma cell lines. Hepatol Res 38: 601-613, 2008.

30. Hirao T, Nanba D, Tanaka M, Ishiguro H, Kinugasa $Y$, Doki Y, Yano M, Matsuura N, Monden M and Higashiyama S: Overexpression of ADAM9 enhances growth factor-mediated recycling of E-cadherin in human colon cancer cell line HT29 cells. Exp Cell Res 312: 331-339, 2006.

31. Mahimkar RM, Visaya O, Pollock AS and Lovett DH: The disintegrin domain of ADAM9: A ligand for multiple beta1 renal integrins. Biochem J 385: 461-468, 2005.

32. Demirkan BM: The roles of epithelial-to-mesenchymal transition (EMT) and mesenchymal-to-epithelial transition (MET) in breast cancer bone metastasis: Potential targets for prevention and treatment. J Clin Med 2: 264-282, 2013.

33. Xie Y, Liu S, Lu W, Yang Q, Williams KD, Binhazim AA, Carver BS, Matusik RJ and Chen Z: Slug regulates E-cadherin repression via p19Arf in prostate tumorigenesis. Mol Oncol 8: 1355-1364, 2014

34. Cui Y and Yamada S: N-cadherin dependent collective cell invasion of prostate cancer cells is regulated by the $\mathrm{N}$-terminus of $\alpha$-catenin. PLoS One 8: e55069, 2013.

This work is licensed under a Creative Commons Attribution-NonCommercial-NoDerivatives 4.0 International (CC BY-NC-ND 4.0) License. 